\title{
Tanja Wiehn
}

\section{(UN)PREDICTABLE ACTS OF DATA IN MACHINE LEARNING ENVIRONMENTS}

\begin{abstract}
This paper investigates artistic representations of machine learning and their interventional potential. Taking its point of departure in two works of art, the paper discusses effects of predictability and unpredictability caused by machine learning systems. By thinking through "eventfulness" (Bucher) and "nonconscious cognition" (Hayles) in human and non-human environments, the paper analyzes the potential of artistic practices to question and rethink algorithmic processing. The paper provides a framework in which artwork challenges forms of technological predictability and comes to terms with machine learning as a fundamental cultural practice in its own right.
\end{abstract}

APRJA Volume 8, Issue 1, 2019

ISSN 2245-7755

CC license: 'Attribution-NonCommercial-ShareAlike'. 


\section{Encounters with machine learning}

Daily entanglement with the technological other has ambivalent results. Concerns about the growing impact of algorithms cast doubt upon objectivity and reliability in systems of machine learning and artificial intelligence, notably when their implementation can have strong societal ramifications (Mackenzie; Wang). Critical investigations are simultaneously emerging in research discourses such as New Media Studies, Internet Studies, and Algorithmic Studies to analyze and question the belief that these technologies are becoming providers of solutions to complex social equations. Algorithmic tools are advocated as means of avoiding all-too-human glitches and forms of unpredictability caused by a subjective human intervention, as they seem to be "stabilizers of trust, practical and symbolic assurances that their evaluations are fair and accurate, and free from subjectivity, error or attempted influence" (Gillespie 179). Yet does algorithmic processing of large amounts of data necessarily guarantee neutrality? Critical investigations of data processing discuss the numerous issues regarding implementation of machine learning and its potential to reproduce racist or sexist biases (Kitchin; Wang). Scholarship has thus begun to look more closely at the notion of the algorithm, the ways in which data is used, and how these relate to machine learning. As a result, scholars are looking into the ramifications of algorithmic decision-making for culture and society, drawing on a diverse set of methodological approaches (Elish and boyd; Seaver; Kitchin; Gillespie). The movement from algorithms towards machine learning tools is of particular relevance here. These are subsets of artificial intelligence and are thus systems that are able to learn and adapt
(Alpaydin; Pasquinelli). Machine learning is basically a form of programming that learns from the data provided. As Adrian Mackenzie points out, machine learning is an accumulation of techniques derived from mathematics (statistics) and computer science and is not a fundamentally new technology (Mackenzie). Machine learning consists of data training, algorithm learning, and model application. Each of these basic components are crucial for generating an output that - to put it very simply - is based on the idea of pattern recognition (Pasquinelli). Moreover, machine learning is already in everyday technological use, operating in the background to recognize faces at border control, to generate credit score rankings, and to provide Facebook's news feed: it is thus more or less visible and tangible (Mackenzie).

A machine learning system is a sort of nooscope, that is a device to map and perceive complex patterns through vast spaces of data - what, in digital humanities, is termed as distant reading. Each instrument of measurement and perception comes with inbuilt and contingent aberrations. As much as the lenses of microscopes and telescopes were never perfectly curvilinear and smooth, similarly Al systems install logical lenses that condense faults and aberrations (Pasquinelli 4).

In contrast to the statistical core elements in machine learning, the perception of the technology itself as a form of artificial intelligence receives a different kind of attention. In their article "Situating Methods in the Magic of Big Data and Artificial Intelligence," danah boyd and Madeleine Clare Elish argue for a reframing of data analysis methodologies that moves away from the terminology of machine learning and towards a computational ethnography. These authors 
problematize not only the faith and reliance in data-driven technologies but also question the seemingly magical moments of artificial intelligence and their branding in commercial environments. They are furthermore referring to uncanny effects caused by machines performing as though they were human. These effects also arise when algorithmic systems perform unintelligible glitches, for instance in showing very poor commercial recommendations or advertisements. However, the algorithmic result can become even more uncanny when the machine is providing results (i.e. predictions) as a basis for decisions that are not as banal or mundane as the display of commercial advertisements. Machine learning-driven surveillance strategies are, for instance, introduced in Western democracies with the claim of offering superior security assessments based on predictable information. Data collection and processing can, for example, become a resource for authorities to assess the likelihood of potential future criminal activities. In her book Carceral Capitalism, Jackie Wang untangles the relationship between algorithms, data analysis, racial discrimination, and their carceral function in the United States. Wang uses the predictive policing tool PredPol to show how data analysis tools are highly reliant on decisions made about their input data. In Wang's example, these human decisions determine the areas and neighborhoods that are under particular investigation. "Although data has been conceptualized as neutral bits of information about our world and our behaviors, in the domain of criminal justice, it is a reflection of who has been targeted for surveillance and policing" (Wang 247-248). Forms of policing informed by machine learning tactics are endorsed as a reliable science, notably for the sake of security. These methodologies are claimed to generate predictability through the collection and processing of data (Elish and boyd; Wang). As noted above, the very composition of machine learning relies upon several factors in which human input and the collection of training data are necessary. As a result, the selection of specific training data shapes the very core of the machine learning model (Mackenzie; Pasquinelli). As Wang notes here, it is always a question of what input data is provided in the training sets.

There is no doubt that algorithmic systems tend to infiltrate and influence more and more aspects of life, in different variations and with varying ramifications. A request for technical insight into these mechanisms seems necessary at this stage, given the problematic outcomes that machine learning environments can entail. Can technical knowledge gain insight into algorithms and their work within machine learning? It is thus far understood that the results of machine learning rely on its various components, including its input data. But how can these systems be understood and managed, considering that they are often described as fundamentally obfuscated and veiled?

\section{The difficulty of knowing algorithm(s)}
Algorithms are inert, meaningless machines until paired with databases on which to function. A sociological inquiry into an algorithm must always grapple with the databases to which it is wedded; failing to do so would be akin to studying what was said at a public protest, while failing to notice that some speakers had been stopped at the park gates (Gillespie 169).

Wang's contribution emphasizes the importance of critiquing the implementation of data-driven technologies. In order to 
formulate such a critique, it is necessary to become knowledgeable about what and how data is collected. Moreover, as Tarlton Gillespie points out in the above quote, the forms of data processing through algorithmic intervention are another crucial aspect. One way of approaching this problem is through a technical understanding of machine learning and the mechanisms that algorithms carry out. Machine learning is highly complex. We have already touched upon some compositions of the technology very briefly here, with a focus on the importance of input data. Matteo Pasquinelli proposes deciphering the training sets and their processing within machine learning. Building upon Kate Crawford's and Vladen Joler's illustration of the Amazon Echo system in Anatomy of an Al System, Pasquinelli highlights the composition and affordances of the different training sets that make the machine learn. He furthermore approaches machine learning algorithms and the production of bias in their outcomes through the statistical and mathematical compositions in place.

By looking at these training sets, it is possible to visualize the human input and decision-making process in machine learning. Taina Bucher likewise highlights the moments of human input in algorithmic processes. For her, these inputs denote a particular interest in the data, the desired outcome and the selection of used data in the first place. The necessary human input in machine learning can thus be characterized by a prior interest in the data, a particular set of assumptions made about a specific case.

A preliminary technical insight is valuable for understanding machine learning as an environment shaped partly by human and partly by non-human agency, as a fundamentally posthuman endeavor (Bucher; Hayles How We Became Posthuman). Investigating this state of entanglement of machine learning practices with culture and their societal aim represents a break from the premature conclusion that automated data processing guarantees reliable information and predictability (Wang). This is what Bucher calls "distribution of agency," acknowledging algorithms as being products of humannon-human environments. "Algorithms are not given; they are not either mathematical expressions or expressions of human intent but emerge as situated, ongoing accomplishments. That is, they emerge as more or less technical/nonhuman or more or less social/ human because of what else they are related to" (Bucher 55). Thinking about machine learning systems as being constructed from distributed forms of agency is helpful for dispelling the idea of objectivity within technology. I'm following Bucher here in considering the embeddedness of algorithms in systems - technological as well as cultural systems. Thus, in looking at the two following works of art, I not only acknowledge the interwovenness of algorithms with cultural practices but also consider these examples as potentially demystifying the magical elements of machine learning as well as forms of human exceptionalism (Hayles).

\section{Machine learning as nonconscious cognition}

In terms of the difficulties of knowing algorithmic processes, I refer to Katherine Hayles' exploration of "nonconscious cognition" to gain entry into the first work of art. In her book Unthought: The Power of The Cognitive Nonconscious, Hayles uses contemporary neuroscience, literary studies, economics, and computer science to work towards the idea of a nonconscious cognition. Hayles describes all forms of a cognition beyond consciousness as the nonconscious. 
She thereby emphasizes the deep entanglements of human systems with technological cognizers. Drawing upon a Deleuzian and Guattarian understanding of assemblage, Hayles develops the term 'nonconscious' in the direction of a cognitive assemblage to account for a wider spectrum of "interactions between human and non-human cognizers" (Hayles 115).

\section{The point of emphasizing noncon- scious cognition is not to ignore the achievements of conscious thought, often seen as the defining characteristic of humans, but rather at a more balanced and accurate view of human cognitive ecology that opens it to comparisons with other biological cognizers on the one hand and on the other to cognitive capabilities of techni- cal systems. Once we overcome the (mis)perceptions that humans are the only important or relevant cognizers on the planet, a wealth of new questions, issues, and ethical considerations come into view (Hayles 10f.)}

Hayles challenges an anthropocentric perspective by deploying the notion of non-human cognizers and is thus in line with other critical posthumanists, like Rosi Braidotti. In exploring Hayles' concept of nonconscious cognition, I will read the following work of art as a cognitive assemblage of a machine learning environment and will attempt to render tangible the intimate entanglement of human and non-human systems. Pandæmonium is an artwork by the Berlinbased duo PWR studio. It is displayed on the website of the Copenhagen-based Annual Reportt exhibition space and was part of an exhibition in January 2018. The piece was not the only work shown in the exhibition but will be the focus here. The artists created an algorithm that runs as a text block down the screen when entering the website. The text block called Pandæmonium involves seemingly meaningful text and, according to the artists, refers to a dream sequence. The composition and visual language of the artwork Pandæmonium allows me to apply the notion of nonconscious cognition to the automated text code shown in the work.

Seen from afar, the piece could at first glance be read as a form of computer code. It is a lively mechanism that is taking over the screen of the device. But this form of a cannibalization of the screen can also be read as a pressing statement of mechanical feelings coming to expression. Pandæmonium's

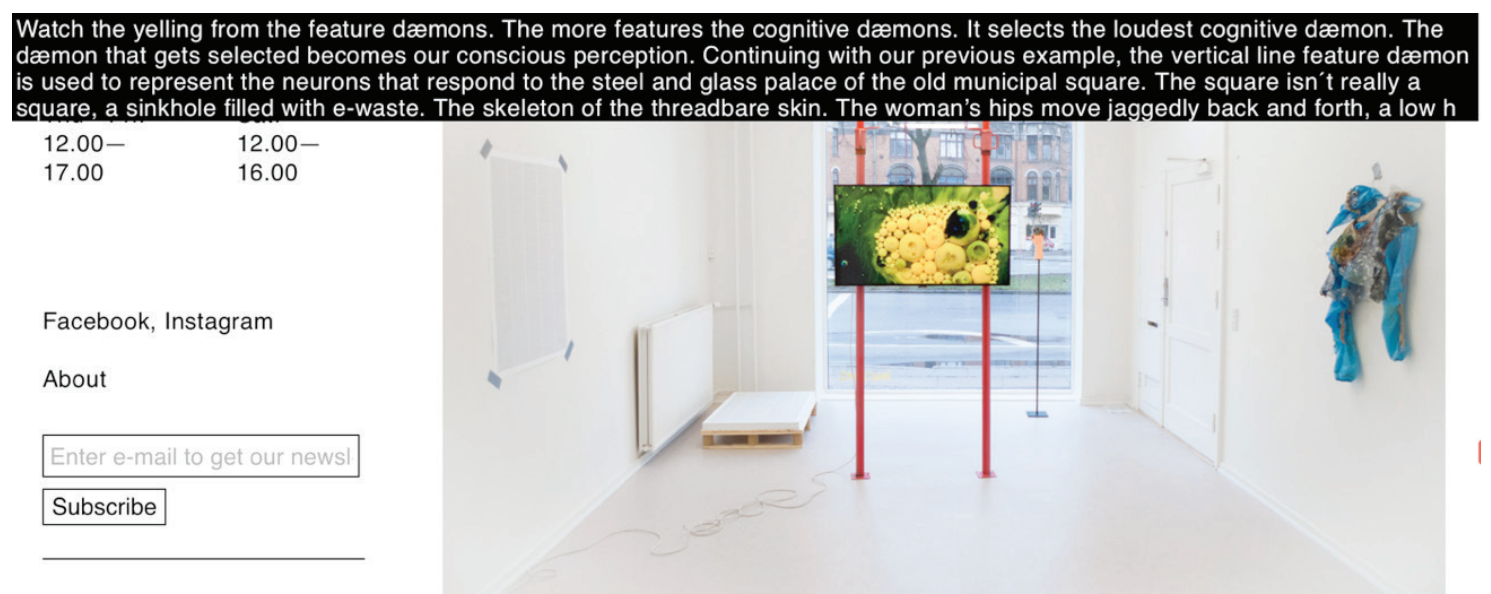

Figure 1: Screenshot from PWR studio's Pandamonium (2018), displayed on Annual Reportt's website, http://annualreportt.com. 
reference to a dream sequence and a nightmare brings opposing notions regarding machine learning to the table. The work produces a layer at the top of the screen, which making its way relentlessly down to the bottom. It adds a code of automated textual information while simultaneously scraping off a layer of the obfuscated and seemingly unknowable machine learning mechanism.

\section{Pandæmonium is a logistical nightmare. Pandæmonium is a pan-computational dream sequence. Pandæmonium shows a future where digital networking has merged with fundamental reality. Everything is an interface to something else. Everything is connected to everything else. \\ Everything is inhabited by autonomous agents acting according to opaque programming (PWR studio).}

The "autonomous agents" mentioned in the above quote evoke the magical elements of artificial intelligence. The provocation lies in part in the machine's ability to produce apparently meaningful text, a fear related to the aforementioned human exceptionalism, the idea of humans being the only creatures to which cognition can be ascribed (Hayles). The visual language of the work displays the hidden and uncategorized feelings of the technological assemblage, simultaneously a dream and a nightmare, both human and non-human. The text block itself entails many references to bones, metacarpal bones, the mouth, the human body and its interaction with an uncertain and ever-changing environment. A close reading of the work's text block might reverse engineer the choices of the categorizations and tokenizing of an input text-corpus. The unintelligible glitches in Pandæmonium, grammatical errors and mechanical failures, have not been erased or corrected. The obfuscated nature of machine learning environments is demonstrated in the glitches and unstructured associations of the work's text code. The text code running down the screen as output becomes the unreadable code that structures the system itself behind the scenes.

In her book If... Then: Algorithmic Power and Politics, Bucher establishes the idea of "eventfulness" within algorithmic procedures. She draws here upon a Whiteheadian notion that focuses on the becoming of an entity, rather than on its simple being. In Process and Reality, Alfred North Whitehead (1978) suggests that the constitution of a being is always related to its process of becoming. Transferring this notion to algorithms, Bucher substantially shifts the question from "what algorithms are to what they do as part of specific situations" (Bucher 49). I thus argue that the progression of the text code, the becoming of the piece of Pandæmonium, fosters this understanding of the eventfulness of algorithms. It is not an end result of data analytics but is a text code in progress, stretching from the top of the page to the bottom. This visual operation of the artwork makes the text block appear as a form of nonconscious cognition. Pandæmonium is becoming, is a form of machine cognition acting out the display of its unexpressed desires. Reading the work through this analytical lens allows us to decipher the basic components of machine learning environments, such as their eventful character (Bucher). The constitution of the work demonstrates the interwovenness of human and non-human cognizers. It is the piece acting as if it were unpredictable, as if it were dreaming and creating unstructured images and thoughts. The work's aesthetics highlight a break from human-centered belief of cognition as exceptionally human (Hayles). 


\section{Material acts of unpredictability}

One of the strengths of - but also one of the problems with - machine learning systems is their mundane yet invisible presence. I noted above Wang's example of their implementation in predictive policing strategies, but they are already in place in services used on a daily basis, such as news feeds on social media platforms (Mackenzie). In these cases, no access is given to how input data is processed or how algorithms are trained. One means of intervening in the categorization and deciphering the seemingly objective predictability in machine learning is through a different kind of engagement with the system itself. The second work to which I refer in this paper fosters ideas of interventions in algorithmic systems through acknowledgement of their embeddedness in a material environment. Through reading the following work, Stop the Algorithm (2018), I seek to rethink unpredictability in machine learning environments as a form of intervention.

The processing and capitalization of affects and attention spans are core patterns used within the big social networks such as Facebook and Instagram. This makes forms of manipulation and control of feelings through data-driven systems difficult to

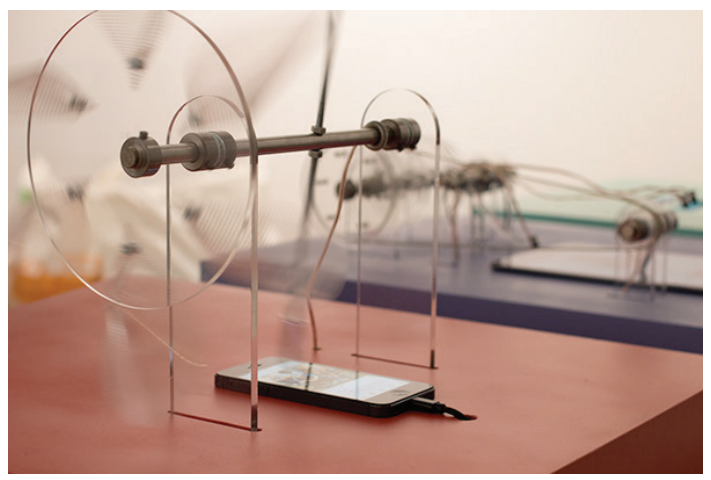

contest, especially in light of the monopolistic power of the big social networks. The artists Stephanie Kneissl and Max Lackner created various gadgets to shift the balance between user and algorithm within the technical assemblage. Their machinic instruments do not actually stop the algorithm, as the name of the artwork suggests, but they change the determination and categorization in the environment of social media sites on both ends: the end of the data input and end of the data output in form of, for example, advertisements shown in the continuous becoming of the newsfeed.

\begin{abstract}
We often assume that those systems are tools, made to connect and inspire us, an infinite playground, an uncontrolled network that constantly reinvents itself. [...] But social media is not neutral but highly biased and has an agenda of its own, with the goal of us to stay online and share as much as possible. What we see on social media is decided by algorithms that are highly subjective, favouring popularity and mass instead of content. This influences our opinions and thoughts. (Kneissl and Lackner).
\end{abstract}

In Fig. 2, a small wind wheel is connected to a pencil. Driven by a ventilator, the pencil swipes through the newsfeed of

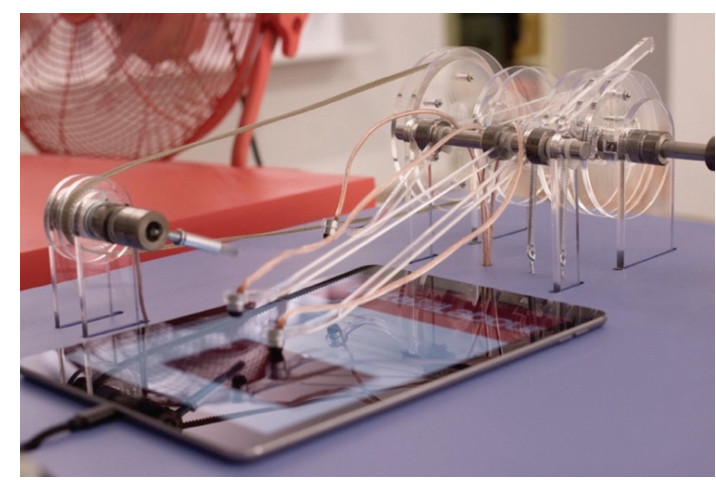

Figures 2 and 3: Stephanie Kneissl and Max Lackner, Stop the Algorithm (2018). 
an Instagram account on an iPhone. In the second gadget shown here (Fig. 3), the pencil is combined with other touching devices. Connected to wheels, they move and stop within the newsfeed - as if something caught their attention - before scrolling further. The movement of the touch on the screen leaves traces of attention within the network and feeds new data into the network. When the little arms touch the surface, they seemingly show an interest in the content, as-if-human. These first two parts of the composition rely on these procedures to maintain a sense of unpredictability: interaction with the physical surroundings take the place of a human cognizer in scrolling through the device.

The artists aim to create random interaction on the basis of material conditions in the exhibition room in order to trick the algorithm. Tricking in this context means engaging with the newsfeed algorithm through an unpredictable method of scrolling. In Stop the Algorithm, the platforms' algorithms are not actually stopped but are instead detrained. The work thus seeks to change one of the fundamental components of machine learning environments.

As the informational networks and feedback loops connecting us and our devices proliferate and deepen, we can no longer afford the illusion that consciousness alone steers our ships. How should we reimagine contemporary cognitive ecologies so that they become life-enhancing rather than aimed toward dysfunctionality and death for humans and nonhumans alike? Recognizing the role played by nonconscious cognitions in human/ technical hybrids and conceptualizing them as cognitive assemblages is of course not a complete answer, but it is a necessary component (Hayles 141).
Rethinking cognition in a post-anthropocentric manner becomes crucial for the second work too. An intervention into closed systems of data harvesting such as social networks cannot be easily realized. The little gadgets in the work substitute a human cognizer within the technological assemblage of this machine learning environment. The artists deploy interaction with the physical conditions of the exhibition room - the wind wheels, scrolling pens, and touching devices on the apparatus - to enable a form of unpredictability. This material interaction is used as a strategy for engaging with and challenging the newsfeed algorithm. The very idea of implementing material elements for creating unpredictability in technological systems is not new however. In cryptography, a distinction is made between true randomness and pseudo-randomness (Gennaro). The latter is called pseudo because it is a mathematically constructed set of numbers so rendered as to appear random. In contrast, true randomness cannot be generated by computers but is often based upon the implementation of a physical, material set of randomness (Doi and Tadaki).

A prominent case is the use of lava lamps in the creation of true randomness in encryption systems. Lava lamps create an environment that coincidentally merges a mixture of oil, water, and wax. By filming the lava lamps around the clock, the internet security company Cloudfare creates true randomness through "the ever-changing

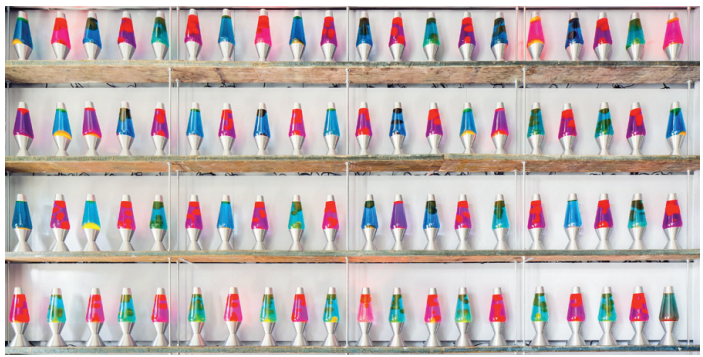

Figure 4: Lava lamp wall at the internet security company Cloudfare, image from WIRED Magazine. 
arrangement of pixels to help create a superpowered cryptographic key" (Airhart). With these measurements, this randomness creates security keys that cannot be predicted by hackers. Only by implementing a material component can unpredictability be introduced. As a result, material randomness holds the potential to overcome the predictability of statistical reductionism in machine learning environments. Simultaneously, the principle of a form of randomness in material environments is becoming an eventful coincidence for a data-driven world. In moments of data processing, these material acts of unpredictability enable a withdrawal from pre-set categorization and classification and debunk the myth of knowledgeable data analytics.

As mentioned above, eventfulness is a core asset of algorithmic environments - from data collection to the learning procedures and training of machine learning that results in artificial intelligence (Alpaydin). The chosen works of art demonstrate an algorithmically and material form of eventfulness that is useful for dismantling myths, uncanny feelings, and magical elements of machine learning as well as for demonstrating their character as technological assemblages of human and non-human environments. In Stop the Algorithm, it is the manifestation of the notion of eventfulness through interaction with a material environment that disrupts the original purpose and capitalization of data collection. Pandæmonium, in contrast, visualizes a process of composition in machine learning.

\section{Conclusion: Machine learning as cultural practice}

There can be no doubting the significance of gaining insight into the technological operations of data collection, databases, and training sets for machine learning. Methodologies of reverse engineering (Bucher), ethnographic research (Seaver), and critical code studies (Cox) possess great potential for constructing knowledge about machine learning technologies. In this paper, I propose including the notion of eventfulness and the idea of nonconscious cognition of human and non-human environments for describing machine learning systems. I believe that these concepts present opportunities for grappling with the potential ramifications of algorithmic processes. They furthermore simultaneously enable the questioning of seemingly objective output and emphasize the necessity of human input. The works of art introduced in this paper help us negotiate these concepts. Aesthetic and artistic representations can contribute to the discourse on machine learning ramifications, highlighting the blind spots of computational determination with reference to algorithms as culture and in culture (Seaver). Moreover, the sustainability of a mere technological insight is questionable in the light of the ongoing development of ever-more complex systems and their interwovenness with capitalist and political structures of oppression and social forms of control (Wang).

The visual language of the works of art provide a framework for expanding a vocabulary of machine learning and introduce creative interventions into algorithmic systems. Pandæmonium questions the meaningfulness of computational processing in the text code that it displays on the exhibition space's website, while the notion of material 
resistance in Stop the Algorithm emphasizes the limits of engagement with highly complex technological systems that cannot be easily decoded or dismantled. The discussion of possible means of engaging with and formulating criticism towards technologies can be accompanied by discursive and aesthetic forms that demystify images of black boxes and debunk the hype of artificial intelligence. Reading machine learning as eventful - as a concept of becoming that entails subjective categorizations and entails nonconscious cognition - transforms technology from being a neutral instrument into a cultural practice. Machine learning systems are meant to guarantee a mode of predictability through the mathematical reduction of complexities. Therein lies potential for the unpredictability of a material embeddedness to recognize the ramifications of machine learning systems and challenge the knowledgeability of their output.

\section{Works cited}

Airhart, Ellen. "How a Bunch of Lava Lamps Protect Us From Hackers." WIRED Magazine, 28 July 2018, https://www.wired. com/story/cloudflare-lava-lamps-protectfrom-hackers/. Accessed 27 February 2019.

Alpaydin, Ethem. Machine Learning. MIT Press, 2016.

Annual Reportt. "Pændamonium." 17. Jan. 2018. http://www.annualreportt.com. Accessed 02 March 2019.

Braidotti, Rosi. The Posthuman. Polity Press, 2013.

Bucher, Taina. If... Then: Algorithmic Power and Politics. Oxford University Press, 2018.

Cox, Geoff, and Alex McLean. Speaking Code: Coding As Aesthetic and Political Expression. MIT Press, 2013.

Crawford, Kate, and Vladen Joler. "Anatomy of an Al system. An anatomical case study of the Amazon echo as artificial intelligence systems made of human labor." 2018. https://anatomyof.ai. Accessed 28 February 2019.

Doi, Norihisa, and Kohtaro Tadaki. "Cryptography and Algorithmic Randomness." Theory of Computing Systems, vol. 56, no. 3, April 2015, pp. 544-80, doi:10.1007/s00224-014-9545-9.

Elish, Madeleine Clare, and danah boyd. "Situating methods in the magic of Big Data and $\mathrm{Al}$, Communication Monographs", 85:1, 2017, pp. 57-80, doi: 10.1080/03637751.2017.1375130. 
Gennaro, Rosario. "Randomness in Cryptography." IEEE Security \& Privacy, vol. 4, no. 2, 2006, pp. 64-67.

Gillespie, Tarleton. "The Relevance of Algorithms." Media Technologies: Essays on Communication, Materiality, and Society, edited by Tarleton Gillespie, Pablo J. Boczkowski, and Kirsten A. Foot, 2014, pp. 167-193.

Hayles, Katherine N. Unthought: The Power of The Cognitive Nonconscious. University of Chicago Press. 2017.

Hayles, Katherine N. How We Became Posthuman: Virtual Bodies in Cybernetics, Literature, and Informatics. University of Chicago Press, 1999.

Kitchin, Rob. "Thinking critically about and researching algorithms." Information, Communication \& Society, 20:1, 2017, pp. 14-29.

Kneissl, Stephanie, and Max Lackner. "Stop the Algorithm." 2018. https://stephaniekneissl.com/reset-social-media. Accessed 02 March 2019.

Mackenzie, Adrian. Machine Learners: Archaeology of a Data Practice. MIT Press, 2017.

Pasquinelli, Matteo. "The Undetection of the New: An Overview of Machine Learning Limitations and Their Social Impact", University of Cambridge, UK, 14 January 2019. Keynote at workshop.

PWR Studio. Artists Webpage: https://pwr. site. Accessed 02 March 2019.
Seaver, Nick. "Algorithms as Culture: Some tactics for the ethnography of algorithmic systems." Big Data \& Society, vol. 4, no. 2, 2017.

Wang, Jackie. Carceral Capitalism. Semiotext(e), 2017.

Whitehead, Adrian North, David R. Griffin, and Donald W. Sherburne. Process and Reality: An Essay in Cosmology. Free Press, 1978. 